\title{
Smart Urban Objects to Enhance Safe Participation in Major Events for the Elderly
}

\author{
Tobias Zimpel, Marvin Hubl \\ University of Hohenheim \\ Stuttgart, Germany \\ Email: \{tobias.zimpel, marvin.hubl\}@uni-hohenheim.de
}

\begin{abstract}
IoT increasingly permeates the public area, e.g., in traffic control and public transport. We propose to equip conventional urban objects with IoT technology to transform them into Smart Urban Objects (SUO's). While there exists some research exploring the potentials, specific solutions to enhance safety for the elderly outdoors are still lacking. The elderly's safety is threatened due to declining physical conditions. As a consequence, the elderly may be excluded from outdoor activities such as participating in major events. Against this backdrop, we design SUOs for adaptive indications of urban hazards, barrierfree passages and for smart reservation of seats to enhance resting possibilities. We report on our solution using Bluetooth technology for remote sensing of older pedestrians serving as input for the objects' adaptive capacities. The SUOs have been installed for test purposes on a major event in a larger German city.
\end{abstract}

\section{INTRODUCTION}

$\mathbf{G}$ ROWING older is-sooner or later-inevitably accompanied by a deterioration of life skills, concerning motor skills, information processing skills and sensory capabilities [1]. This regularly intensifies the individual perception of threats to safety, particularly outside of the own home. Changes in body mechanics and impaired endurance pose a substantial risk for safe mobility [2], [3]. As a consequence, the elderly tend to avoid going outdoors without active assistance. This may lead to declining cultural and social participation up until the feeling of isolation. Empirical research has broadly studied and confirmed the positive influence of outdoor activities for the elderly's well-being [4], [5], [6] and we suggest that IT use of the elderly can have a positive impact on their participation in outdoor activities [7].

Demographic projections foresee a disparity between younger people who can provide care, and older people who will potentially be in need for care [8]. This anticipation virtually reinforces the requirement to find innovative means for assisting the elderly in their outdoor activities up until old age. The role of the built environment in this respect has long been acknowledged [9], [10]. As particular assistive means in the built environment we design so called Smart Urban Objects.

Smart Urban Objects (SUO's) are urban objects equipped with sensors, actuators and enabled to make potentially use of digital information processing. Examples of such SUOs are "smart" park benches, street lights, information panels or parking lots [11], [12], [13], [14]. By interconnecting them via internet technology they are IoT objects.
Unlike the example SUOs, we specifically aim at enhancing safety of older pedestrians for the participation in public outdoor major events. These events can be particularly exhaustive and hence hazardous for the elderly because of several reasons:

On major events use to be a relatively large crowd of people on a limited space. Additionally, the event area is usually characterized by temporary installations. These installations are furthermore rarely tailored for accessibility. All this may lead to increased confusion respectively to increased mental exhaustion and to increased physical exhaustion.

The arrangement of temporary objects also produces unexpected risks of tripping, as for example with cable bridges. Because of the temporary character, people often do not exactly know, where they need to go, if they search something particular. However, even if people know where they need to go, they are often unaware about accessible paths on the temporarily arranged area.

Older pedestrians may have increased need for seating rests. One reason is the aforementioned mental and physical exhaustion but can also be due to dizziness. Weather, especially heat, may be a factor, too. Older people often have impaired thermoregulation because of reduced fluid balance[15], [16]. This may stray the circulatory system even stronger, leading to seating rests for its relief and recovery.

Therefore, we design SUOs that adaptively warn for potential tripping hazards, indicate accessible paths and allow for reservation of seats from anywhere on the area. We have prototypically tested the SUOs on an outdoor public major event in a larger German city.

We make use of Leveson's conceptualization for safety engineering [17]. Safety is constituted as avoidance of accidents where the concept of accidents encompasses all situations that involve some unacceptable loss [17, p. 181]. In this respect, we focus on conditions potentially leading to accidents. Therefore, the concept of hazards is pivotal and is defined as "[a] system state or set of conditions that, together with a particular set of worst-case environmental conditions, will lead to an accident (loss)". [17, p. 184]

On this basis, we make use of the implication that accidents occur only if an hazardous state coincides with some worstcase environmental conditions. Note, that hazards and environment constitute a dualism. That means whether a state is hazardous and whether an environmental condition is a worstcase one is mutually dependent. As our design object is the 
urban environment through the means of SUOs, we define older pedestrians as the "systems" which can potentially be in an hazardous state.

We assume older pedestrians to be in an hazardous state if (a) they are only able to lift their legs comparatively little, (b) have impaired eyesight or (c) are in need for a rest. Corresponding worst-case conditions are (a) structural barriers, (b) "hidden" stumbling blocks and (c) missing seat opportunity. (a) Structural barriers, like curb stones or steps, may lead to an accident, if an older pedestrian cannot lift her legs high enough. (b) Stumbling blocks may lead to an accident if an older pedestrians cannot recognize it visually. (c) A need for a seat rest may lead to an accident, if there is no seat available.

In turn, (a) inability to lift the legs high is no problem if there are no steps or the like, (b) visual impairments do not lead to accidents if there is no hidden stumbling block on an older pedestrian's path and (c) need for a seat rest is not critical if there is an available seat possibility. Conversely, if (a) a pedestrian can lift its legs high then steps are no effective barriers, if (b) stumbling blocks are visually recognized, they pose only little risks and if (c) a pedestrian has good endurance, missing seats are no safety problem.

This shows that principally two options are possible for enhancing safety: Avoiding hazardous states or avoiding worstcase environmental conditions. Since in our conception the hazardous states are inherent to the older pedestrians, we take them as given and seek to avoid the corresponding worst-case environmental conditions.

(a) The disability to lift the legs appropriately must be taken as is. However, the corresponding worst-case environmental condition can be avoided by guiding the pedestrians through passages without steps, curb stones and the like. Then, on the pedestrian's individual path, there is no worst-case environmental condition. (b) Impaired vision must be taken as is. However, the corresponding worst-case environmental condition can be avoided by clearly indicating stumbling blocks, thus "unhide" them. There, we expect that an adaptive indicator is more salient than a static indicator. (c) Need for a rest, eventually, is taken as given. However, by reserving some seats and making them available adaptively to older pedestrians who announce a need for a rest, the worst-case condition that there is no available seat can be avoided.

Against this background we formulate our design-oriented research question:

\section{How to design Smart Urban Objects (SUO's) for major events to adaptively avoid worst-case environmental conditions for older pedestrians being in a defined hazardous state?}

This paper proceeds as follows: In section II, we review the state of the art on IoT conceptualization as well an on pedestrian support with smart objects. In section III, we report on the design of our SUOs. In section IV, we evaluated our SUOs in terms of its principal functionality. In section $\mathrm{V}$ we discuss our SUOs and revised some conceptional issues, like IoT. In section VI we conlude our work and provide an outlook.

\section{StATE OF THE ART}

\section{A. Internet of Things and Smart Objects}

Most basically "IoT is the network of things, with device identification, embedded intelligence, and sensing and acting capabilities, connecting people and things over the Internet." $[18$, p. 4] As IoT objects are characterized by their situatedness in a physical real-world environment, sensors provide an input interfaces from the environment to the IoT object and actuators provide an output interface from the IoT object to the environment. Sensors convert physical signals from the realworld environment into digital data. Actuators convert digital data into actions that shall affect the environment.

In our conception, we allow that sensory input can be digitally pre-coded data, as for example when sensing radio signals, such as RFID or Bluetooth signals. While actuators are often considered to be physically moving parts [18, p.71], we include in our conception also non-moving parts that shall exert influence on the environment, like lights, audio-output, displays.

IoT can be seen from three perspective [19]: (a) The "Things"-oriented view contains technologies such as RFID, UID, wireless sensors and actuators as well as that the things shall be abele to communicate with each other [19]. (b) The "Internet"-oriented view containts technologies such as IP for Smart Objects (IPSO) or Web of Things as well as middleware [19]. (c) Additionally a "Semantic"-oriented view can be taken, containing semantic technologies, e.g. for reasoning over data [19].

IoT conventionally has four layers [20]: (1) The sensor/actuator layer relates directly to the sensors, actuators as well as to the IoT objects themselves, hence to hardware [20]. (2) The network layer relates to the basic network technologies for data transfer [20]. (3) The interface layer provides methods for interactions with the IoT objects for other applications and users [20]. (4) Finally, the service provides services to satisfy user requirements [20]. This means, applications can be abstracted from the hardware-oriented sensor/actuator layer and be implemented on the service layer.

IoT objects are often referred to as smart objects. There are several differentiations for the "smartness" of the objects. One attempt is to differentiate the awareness capabilities [21]: (1) Activity-aware objects understand the environment as events that are directly linked with the object, such as touching the object [21]. (2) Policy-aware objects relate events to organizational policies. (3) Process-aware objects relate events to organisational processes [21]. Although this differentiation seems to focus on applications for business operations, it is conceptually applicable to other applications, e.g. if organizational policies are substituted by other norms.

An alternative typology of smart objects is given by their (I) capacity to store relevant data, including an identifier for themselves, (S) capabilities for sensory perceptions and (A) execution of actions with actuators, (D) decision-making 
ability and finally $(\mathrm{N})$ network connectivity [22]. Referring to the letters in brackets, an "I-N object" for example has an identity and data storage (where at least its identity is stored) and network connectivity. Note that not all combinations are considered to be realistic as for instance most object types without "I" [22]. Note also that objects need to implement a form of advanced information processing to exhibit decisionmaking abilities.

Smart objects are an important information technological basis for Smart City [23] as an application of IoT [24], [25]. Most conceptualization of Smart City contain or even accentuate inclusiveness as an aim. In this respect, we consider our IoT application to be a Smart City use case.

\section{B. Smart Urban Objects and Pedestrian Support}

Poulsen et al. propose an urban light system that can response adaptively to pedestrian's occupancy patterns, wind velocity or that can be customized to individual color preferences via smart phone [12]. Albeit not in an outdoor setting, the potential effect of adaptive colors on the mood of seniors has been studied by Huldtgren et al. [26]. Cunha \& Fuks propose to use light systems as a "host" for sensors to support continuous proactive care within a feedback loop [27].

Another type of objects are public interactive screens. These can be utilized as adaptive urban information panels. Cremonesi et al., Müller et al. as well as Vogel \& Balakrishnan examine concepts for personalized interactions on such public screens [13], [28], [29]. The basic approach is to define virtual fences around the screen and for identified pedestrians in near proximity sections on the screen can be personalized.

As an interesting application that is more directly directed towards pedestrian support, Traunmueller \& Schieck introduce a so called space recommender system [30]. There, routes can be recommended individually based on recommendations of other pedestrians concerning their walking experiences. While this system does not constitute a SUO in the narrower sense, it still can serve as a useful complement system, possibly running on public screens as recommendation input device.

Concerning public transport experience, Foell et al. propose an IoT based system for so called disadvantaged users [31]. "Disadvantaged" users are novice users, tourists, people with handicaps and older adults who have difficulties in orientating themselves properly in an unknown or uncomfortable environment. The system provides support in so-called micronavigation, e.g. whether a person is in the correct bus or in how many minutes she needs to get off [31].

For supporting pedestrians with impaired vision Kumar et al. propose an assistance system that can run on a smart phone to detect obstacles and recognize the faces of acquaintances [32]. Although, this system shows similar problem solving structures as our system has, we rather focus to make the urban objects smart in the sense of interaction end devices.

\section{ARtifact Design}

To transform urban objects into SUOs we design a system against the following requirements: It must include

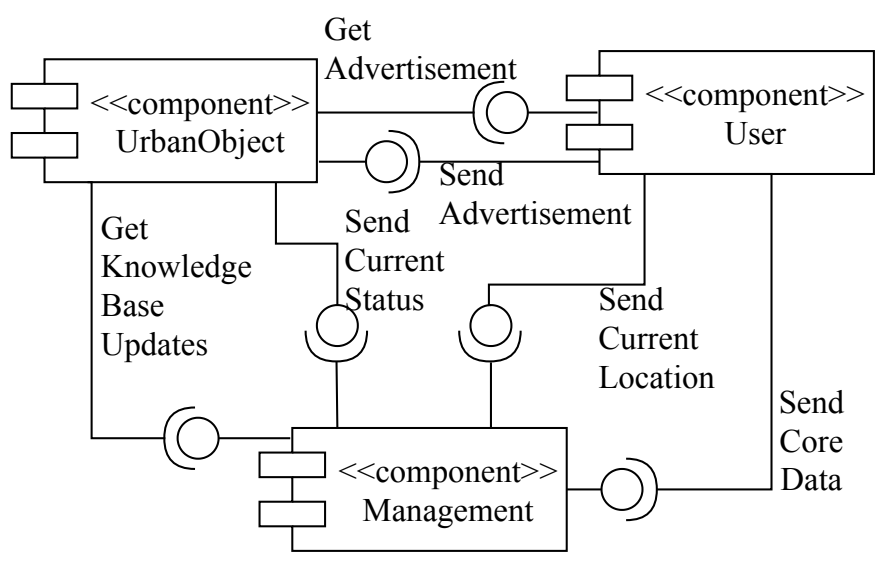

Fig. 1. Architectural Overview

1) the ability to book seats.

2) navigation information.

3) adaptive indications.

(1.) The ability to book seats is required to enable smart reservation of seats. (2.) Navigation information is required to guide older pedestrians via barrier-free passages. (3.) Adaptive indications are required to warn for urban hazards.

Fig. 1 shows the overall architecture of our whole system, including components for older pedestrians (User component) and SUOs (UrbanObject component). The SUO executes the UrbanObject component on small attached computers (e.g. "Raspberry Pi"), while mobile devices of older pedestrians (such as "Android" phones or "iPhones") run the User component to allow booking requests and control Bluetooth signals. To coordinate SUOs in the overall system, we use a scalable central unit (Management component, see Fig. 2), based on service-oriented architecture. We use Secure Sockets Layer connections between each component and a protected database to provide basic security. Our Management component mainly consists of three sub-components-a seat management component (Booking component), urban object component (SmartObject component) and real time data processing component (Live component) for sensor data (e.g. current location of an older pedestrian or park bench temperature).

\section{A. Management Component}

Each User or UrbanObject component manages only a subset of knowledge and cannot share knowledge with other User or UrbanObject components. Our Management component, therefore, provides services in distinct components to coordinate older pedestrians and SUOs and act as a global knowledge base. The following specialized components provide these services:

- Booking: Allocates a booking request to the best-suited seating accommodation with free seats.

- SmartObject: Provides knowledge and services for SUOs.

- Live: Integrates heterogeneous data from SUOs or devices of older pedestrians and updates the global knowledge base in real time. 


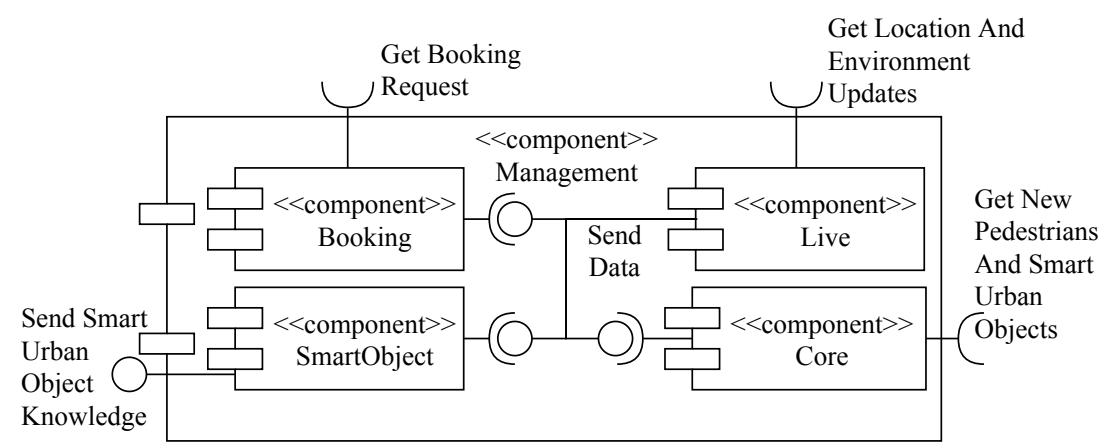

Fig. 2. The Management component

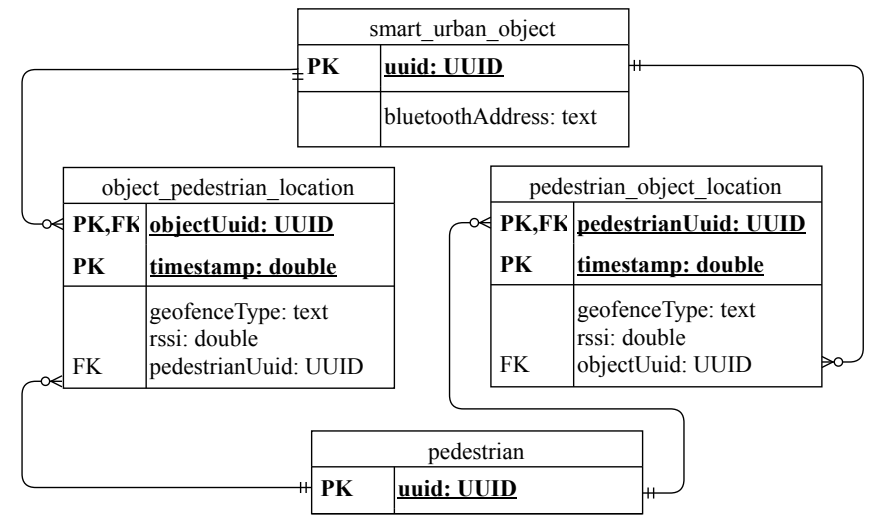

Fig. 3. Section with different primary keys

- Core: This component manages relatively static data such as identifiers for an older pedestrian or properties of SUOs (e.g. seat capacity).

Each of these specialized components provides REST-APIs for the User or UrbanObject component. Both components using HTTP requests to consume these REST-APIs. Hence, a network connection between components is necessary. Each component uses interchangeable data objects if they use the same data object. We're using an additional background service to manage load balance across multiple component instances and detect component failures. This background service registers all management component instances.

The interaction sequences take into account two different but simultaneous views. The first view considers the sequence from the perspective of the SUO, whereas the second considers the perspective of the older pedestrians.

\section{B. Interaction Sequences}

The SUO sends its current status to the UrbanObject component and waits for the reply (see Fig. 4). Subsequently, the SmartObject component queries seat bookings and determines the most relevant information. This information consists either of the next seat booking on the SUO or of individual routing information for an older pedestrian. If the SUO has pending bookings, they will be transmitted, otherwise routing information for the nearest located older pedestrian. Then, the SUO can interact with the older pedestrians, e.g. display information or adaptive indications. While older pedestrians moving through the major event area, their device uses Bluetooth Low Energy network technology to detect SUOs based on Bluetooth addresses (see Fig. 5). Subsequently, their device sends continuously RSSIs (Received Signal Strength Indicators) and Bluetooth addresses of detected SUOs to the Live component. Each SUO advertises Bluetooth Low Energy services with a static Bluetooth address. Based on the RSSI the Live component approximates the location of the older pedestrians, detects location zone changes (e.g. leaving a seat) and updates the global knowledge base. Simultaneously, the device of an older pedestrian advertise nonexistent Bluetooth Low Energy services at regular intervals (see Fig. 6). The device introduces a temporary service based on a new service identifier and a random Bluetooth address. This temporary service is not connectable for other Bluetooth devices. Meanwhile, SUOs listen to new Bluetooth Low Energy services and show adaptive light indications on urban hazards. The indication intensity depends on the highest service RSSI gathered from a listener for new service detection. When an older pedestrian approaches the SUO, visual indication on urban hazards increases. The advertisements and scans on the device of older pedestrians are independent of whether the User component is in the foreground or background.

\section{Live Component}

SUOs and devices of older pedestrians transmit in an interval of one or two seconds, information about their environment or location. Each location consists of the recognized object and RSSI value, a timestamp and the corresponding older pedestrian. As a consequence, each location gets assigned to one SUO. The Live component aims to provide information to other components as quickly as possible. To avoid query join operations and time-expensive where conditions we store time-sensitive information of older pedestrians (e.g. their location or bookings) in redundant tables. Each time-sensitive information table has either the nearest SUO or an identifier for the older pedestrian as part of the primary key. Our primary key is complemented by a timestamp to read the newest insertion first, without additional sorting. Fig. 3 shows a section of our database schema providing these characteristics for 


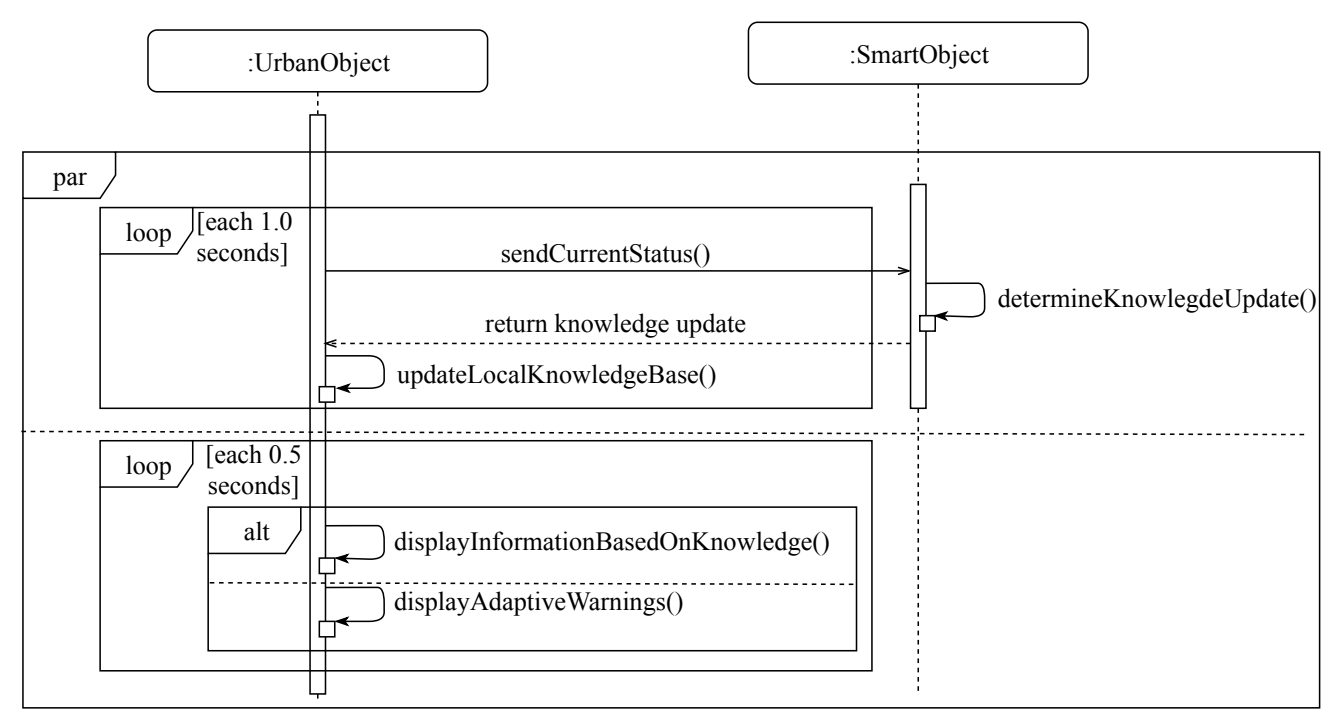

Fig. 4. Interaction between SUO and UrbanObject component

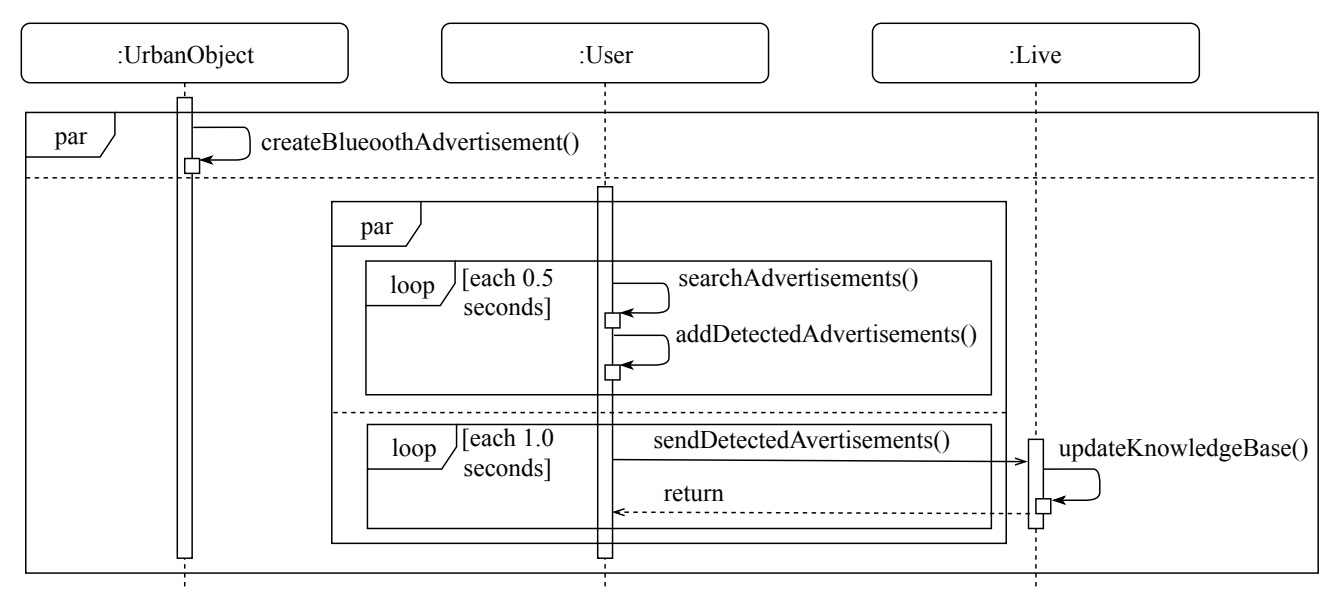

Fig. 5. Interaction between older pedestrian, SUO and real-time processing component

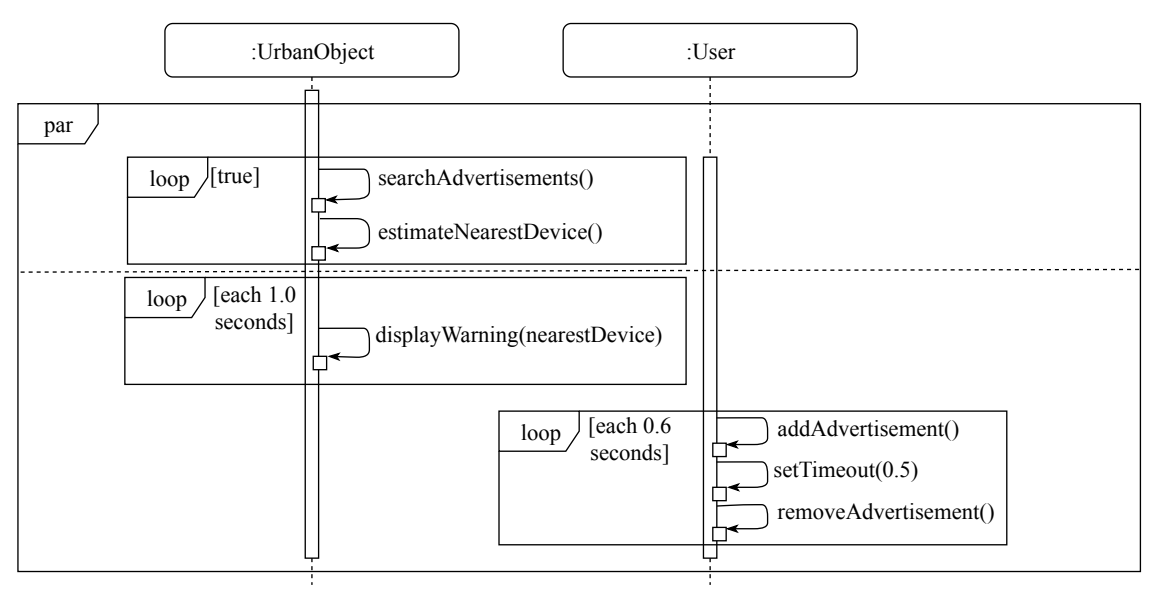

Fig. 6. Interaction between older pedestrian and SUO 
locations. Due to the expected amount and frequency of data, we use a distributed database management system with twodimensional key-value tables. Before providing information, we use gathered information to update possible dependent aspects, like the completion of bookings.

\section{SmartObject Component}

The UrbanObject component only receive required information for the estimated time between knowledge requests to limit resources (e.g hardware, web or computation). For this reason, the SmartObject component provides individual knowledge base updates for UrbanObject components. Its main goal is to select the most relevant information, composed of bookings on the own device or routes. We prefer information about bookings on the object to routes to other destinations for park-benches (see algorithm 1). The algorithm updates the new knowledge base with booking information of any nearby older pedestrian with a newer location update than the latest knowledge base update. If there is no older pedestrian with booking for the requesting object, our algorithm uses routing information to a booking for one older pedestrian with the latest position update as a knowledge update. To force the UrbanObject to show adaptive indications instead of booking or routing information, the SmartObject returns an empty knowledge update. In contrast to an empty knowledge update, we can force the UrbanObject to display independent pedestrian information by simulating bookings and locations.

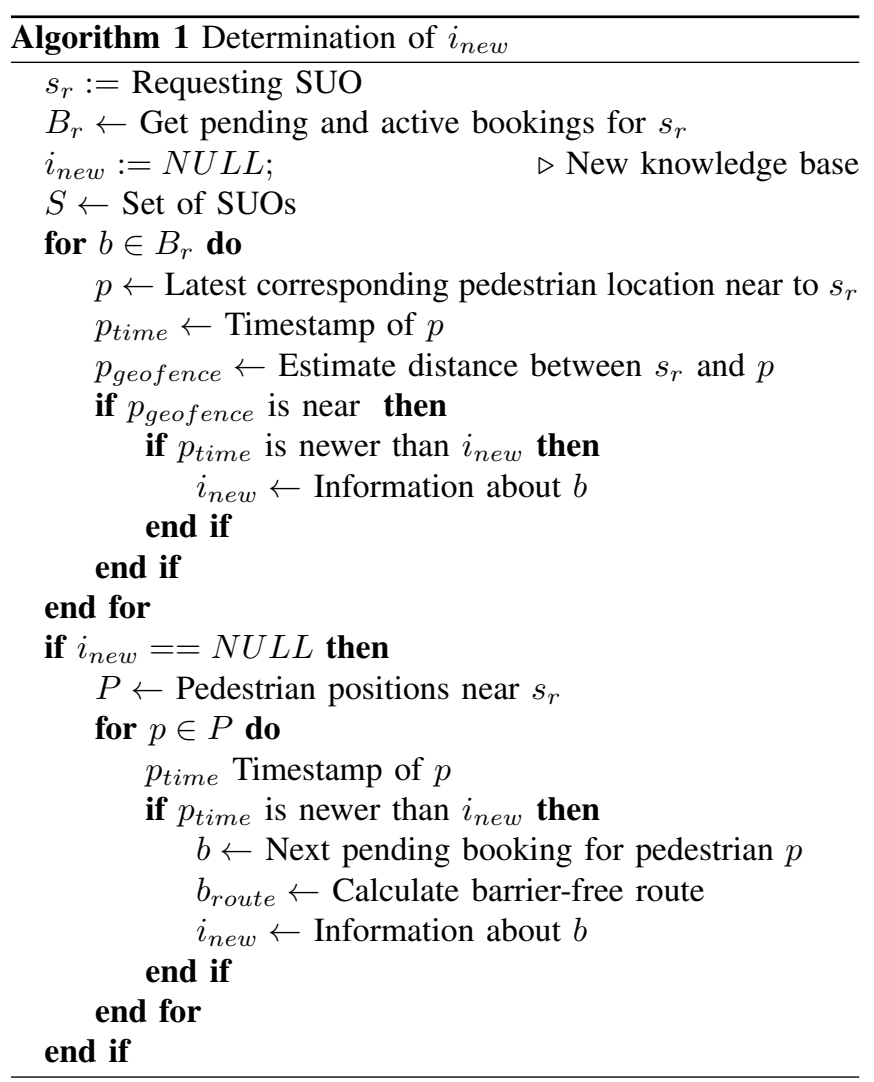

We approximate the distance between an older pedestrian and SUO with the obtained Bluetooth RSSI. Algorithm 2 converts the Bluetooth RSSI into an absolute number. Then, the algorithm divides the absolute number into geofences with weighting, whereby a lower geofence weight indicates a shorter distance. We use the geofence weight as distance lower bound between an older pedestrian and SUO. We use the SUO geolocation to determine pedestrians geolocation. Hence, the SUO geolocation corresponds to pedestrian geolocations if the approximated distance is close enough. Otherwise, we can't determine pedestrians geolocation.

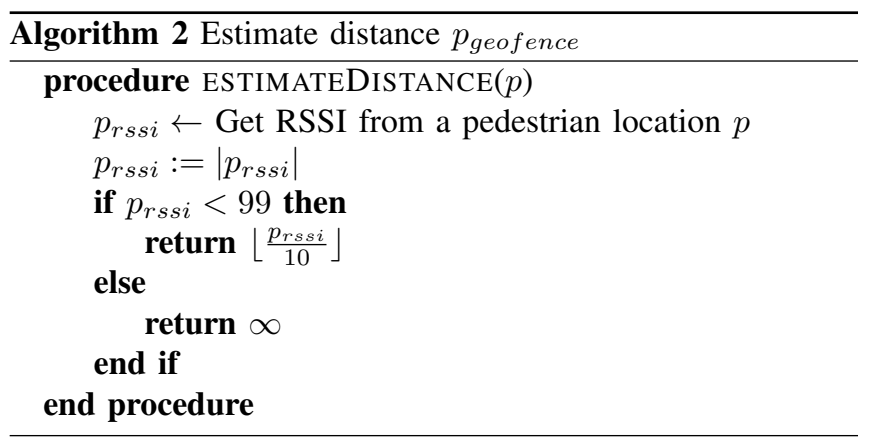

\section{E. Booking Component}

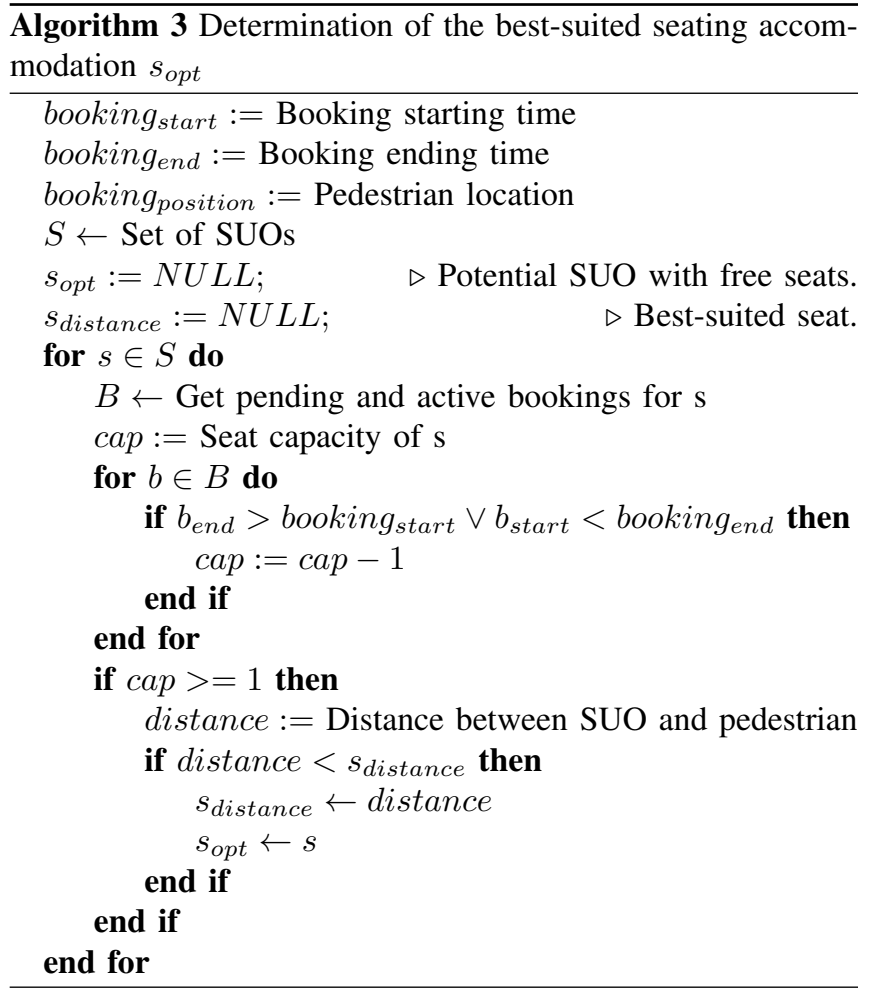

The booking component is responsible for seat management and allocates seat preferences of older pedestrians to park benches. Its main goal is to achieve the best possible seat allocation for each older pedestrian based on their location. In order to achieve this goal, the system has to perform 
two main tasks: monitoring of seats for occupancy detection and identification of the older pedestrian. It therefore uses the collected and processed data about detected SUOs by devices of older pedestrians (location of the older pedestrian), as well as information about the environment of seating accommodations. Algorithm 3 shows the allocation algorithm using environment and information of older pedestrians. The algorithm validates for each pending and active bookings of all seating accommodations if any seating accommodations have seats for the booking request. We calculate the number of free seats for one seating accommodations through subtraction of reserved bookings in the corresponding time slot from the individual total number of seats. This time slot starts five minutes and ends 30 minutes after the booking request. Thereby, this results in a 30-minute seat booking. We use the estimated distance between older pedestrians and seating accommodation as decision base and prefer a short distance. Therefore, our algorithm provides the nearest free seat accommodation. Subsequently, we transmit the allocated seat to our Live and User component to enable routing and provide visual feedback. If the older pedestrian arrives his booked park bench and her User component recognizes the seating accommodation at least two times, we mark the booking as active. We mark the seat as free if the older pedestrian leaves the seating accommodation or his booking end occurs. The older pedestrian leaves the seating accommodation if her latest obtained location is in an outer geofence zone for this seating accommodation.

\section{F. Core Component}

Before interacting with older pedestrians, the User and UrbanObject components need to register once and become known to other component instances. Hence, the Core component creates a unique random identifier for each new User or UrbanObject and informs the Live component. Another responsibility for this component is the attribute management for different kinds of SUOs. The UrbanObject component provides additional attributes to identify it in case of downtime, whereas the User component identifies itself (e.g. name or geographic coordinates). If any User component can not identify itself, we consider this User as new User component. Further attributes for components depend on the corresponding object type. This includes seat capacity for SUOs with type seating accommodation, whereas the type for urban hazards include different indication types.

\section{G. UrbanObject Component}

The UrbanObject component on the SUO control detection of an older pedestrian and provide visual feedback for the older pedestrian. Therefore, we connect the component with a color display and Bluetooth Low Energy Module. We overwrite obtained knowledge from SmartObject component and recognized older pedestrian if newer knowledge is available. If knowledge is available, the UrbanObject component can show this knowledge. Due to random Bluetooth address from mobile devices of older pedestrians, we aggregate recognized older pedestrians. Then, we submit this aggregation as status to Live component. If the component shows an adaptive indication, we transform the highest Bluetooth RSSI within two seconds into a percentage value and use this value as indication intensity. In case of a difference greater ten between the last and current percentage value, we use the average as indication intensity for a smooth transition.

\section{H. User component}

Apart from a user interface to request a seat, the User component is responsible for the localization of an older pedestrian. Localization consists of scanning for SmartObject components and advertising of own services. This component compares detected Bluetooth devices against Bluetooth addresses in its knowledge base and filters UrbanObject components. Due to possible operating system restrictions from mobile devices of older pedestrians and changed settings, the User component has to monitor the outcome of localization operations. If the User component detects any deviation, it pauses the concerning operation, until the user solves it. We store the unique random identifier for an older pedestrian on their mobile device to keep the same identifier and allow component shutdowns and restarts. Thus, after a restart, it starts automatically scanning and advertising. If an older pedestrian rejects the localization, he enables an incognito mode, where his device advertises services for adaptive indication but do not scan for UrbanObject components. Consequently, no localization information will be transmitted. The mobile device has to support Bluetooth Low Energy to use the overall system.

\section{Functional Testing}

To show the feasibility of our artifact, we conducted two scenarios, addressing different safety aspects. The first scenario, shown in figure 7 , addresses the booking of resting possibilities to counteract against exhaustion. In contrast to the first scenario, scenario two (see fig. 8) focuses on the prevention of risks from urban hazards by adaptive indications. Scenario "seating accommodation" covers the need for seats based on exhaustion or physical restrictions of an older pedestrian if elderly recognize their need. Then, they announce their need for a seat, in this case, to our artifact. Our artifact will search for the best-suited seat, books this seat and informs sub-components. Subsequently, the older pedestrian receives her booking and goes to her seat. On the way, she sees routing information from UrbanObject components that allows her to find the barrier-free way to his seat. When arriving, the seat shows that the older pedestrian reached his destination.

The second scenario "adaptive indication" shows indications in different intensity based on the estimated distance between urban hazards and older pedestrians. On the other hand, if the older pedestrian walk away, the indication intensity decreases.

We suppose our artifact to be feasible if we provide technical functionality that is required to enable seat bookings for resting possibilities and adaptive indications on urban hazards.

Therefore, our artifact has to approximate the distance between the older pedestrian and urban hazards, estimate the 


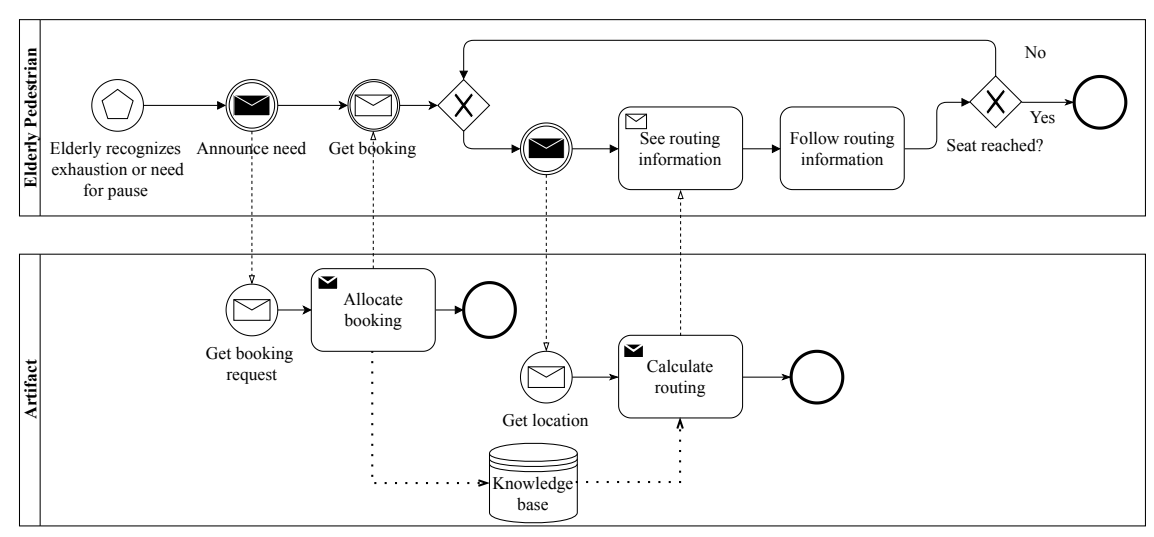

Fig. 7. Scenario "seating accommodation"

location of the older pedestrian and distribute knowledge. We validate the location detection and knowledge distribution in scenario "seating accommodation" and use scenario "adaptive indication" to prove the approach detection.

Our scenario-based testing takes primary place on the property of our institute. However, we have been able to reproduce the settings at the "Turmfest Rheydt 2019", a major event in a larger German city. The settings include Raspberry Pi's with LED matrices, Android phones, a laptop and a router. We have positioned Raspberry Pi's at least five meters apart. Each Raspberry Pi runs the UrbanObject component with a preregistered SUO, while the laptop executes the Management component with sub-components. Android phones run the User component that includes a preregistered older pedestrian. Each device connects to a private wireless network, made available by a router, to consume REST-APIs. We implement the UrbanObject component in Python, whereas the User component is in Typescript and the Management component in Java. Our Management component uses Apache Cassandra as database system. In the study are other Bluetooth Low Energy devices, such that UrbanObject components started to run two minutes before starting each scenario.

In scenario "seating accommodation", the Raspberry Pi's represented seating accommodations. We requested seats via the User component, while we stayed outside the detection range of all Raspberry Pi's. Then, we got a seat allocation and started to walk to a component, which was not our allocated seating accommodation. The UrbanObject component on this Raspberry Pi displayed an arrow towards our allocated virtual seating accommodation within five seconds. Meanwhile, we went further to our allocated seating accommodation, that displayed a symbol that represents a free seat when arrived in a zone of five meters. If entered a zone of a half meter and allocated seating accommodation, the component displays another different symbol within ten seconds. The scenario shows that our artifact can estimate locations of an older pedestrian and distribute knowledge across multiple SUOs. However, there can be a time gap of five to ten seconds between arriving and detection of arriving.

In contrast to the scenario "seating accommodation", in scenario "adaptive indication" our Raspberry Pi's simulated urban hazards. Again, we started outside the detection range of all Raspberry Pi's, so no indication was displayed. We went in walking pace straight towards a Raspberry Pi. The indication appeared during the movement and the warning intensity increased with decreasing distance. Finally, the indication disappeared. However, the indication intensity jumped at the same distance. We find the Bluetooth RSSI of the Android phone changed in a range of plus-minus six at the same distance. In fact, the scenario "adaptive indication" demonstrates our artifact provides approach detection. Nevertheless but we can not provide the exact mapping from Bluetooth RSSI to distance. Based on findings of scenario "seating accommodation" and scenario "adaptive indication" we suppose the feasibility of our artifact.

\section{Discussion}

We contribute a system to enable adaptive indications of urban hazards, barrier-free passages and smart reservation of seats. Thus, we transform urban objects into connected SUOs. We described two scenarios where SUOs support elderly to participate in major events. Our evaluation does not apply to adapted scenarios that include shielding elements or smaller distances between SUOs. In contrast, our evaluation applies to scenarios in urban areas or with an increased number of SUOs. We did not consider or compare the signal strengths of different small computers (inclusive accessories like displays) and mobile phones, including their operating system. Findings concerning the processing or handling of volatile Bluetooth RSSI shows white spots for further research. The artifact extension of localization with GPS-geographic coordinates may be future work. Due to our centralized approach, scalability limitations may occur. As a consequence, we can distribute our database about multiple machines or instantiate multiple component instances to counter.

Since our artifact does not require any special characteristics of the elderly and is not tied to any position or event type, our artifact can be used by all pedestrians and in all urban areas. The smart seat reservation including routing via barrier-free passages corresponds to routing from one location to another. 


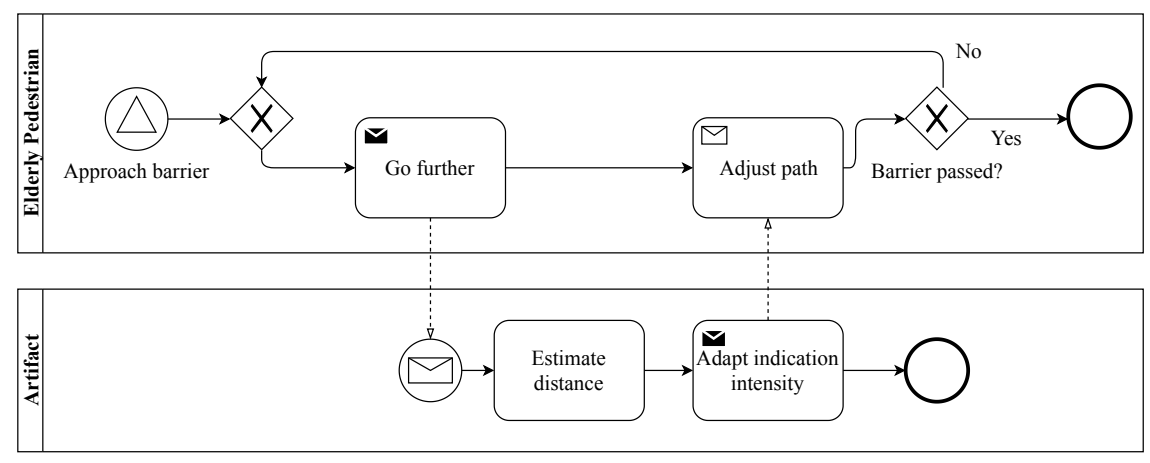

Fig. 8. Scenario "adaptive indication"

In particular, people with a handicap can benefit from our artifact, too. Included functions in our artifact are transferable to other uses cases. For example, the smart reservation of rest possibilities can constitute a basis for individual routing to and reservation of toilets. Another application may be the smart reservation of parking slots (including routing) or the barrier-free finding of dining possibilities and dine pre-order for the elderly for major events. In addition, our artifact can support pedestrians in their everyday lives. The consideration of environment data (e.g. the number of people in a particular area) for routing calculation may be future work too.

Regarding our understanding of sensors and actuators explicated in section II, our SUOs can be classified as I-S-A-N objects according to the typology of López [22]. The SUOs have a unified identifier and some data storage (I), are able to sense the environment through Bluetooth signals (S), can affect the environment with visually outputs from the LED displays (A) and are connected in a network (N). For our purposes, this network can be a local area one. However, connecting the SUOs to the internet would be conceptually equal. Decision-making ability (D), that goes beyond simple stimulus-response, is delegated to the central management component, cf. figure 2. This management component implements the service layer in the sense of Xu et al. [20], as described in section II, too.

Revisiting our research question, table I summarizes how our SUOs can contribute to safety. The safety-engineering approach guided our pre-design phases with the conceptual separation of hazards and worst-case environmental condition. By "accepting" that we cannot alter some circumstances concerning the pedestrian state or the environmental conditions, we focused on means for avoiding that worst-case environmental condition coincide with an older pedestrian being in a defined hazardous state. Therefore it is of less importance whether the pedestrians are defined to be the system or the SUOs than to actually define respective states and conditions. These need to be defined in a way such that an engineer gets a "point of attack". When a hazard is given, the engineer can try to find a way, how the environment can be adapted with feasibly means. When an environmental condition is given, the engineer can try to find a way, how the system resp. pedestrian state can be adapted, if possible. As adaption of the pedestrian state is seldom possible, a feasible way is then to find means that the pedestrian avoids sub-environments with worst-case conditions. Quite obviously, in our case we could also have referred to worst case environmental conditions as hazards in the environmental subsystem. We decided to define the pedestrians to be the system because our explicit approach is to make the environment adaptive to the pedestrians requirements.

\section{CONCLUSIONS}

We proposed Smart Urban Objects (SUOs) for (1.) adaptive indication of accessible passages, (2.) adaptive indication of stumbling blocks and for (3.) reservation of seat on public outdoor major events. Example scenarios are respectively:

1) An older pedestrian approaches a step but cannot lift her legs high enough to safely pass it. The step as an SUO detects the older pedestrian and points in the direction of an accessible passage with a lower step or no step at all.

2) An older pedestrian approaches a "hidden" stumbling block, like a small polder. The polder as an SUO detects the older pedestrian and warns her visually. Thereby we expect that an adaptive warning will be more salient and hence contributes more perceived safety of older pedestrians than a static one, which is always visible.

3) An older pedestrian needs a seat rest. She can reserve a seat from anywhere on the area. When she passes another SUO, the display of the SUO shows a pointer in the direction of the reserved seat. When the older pedestrian approaches the reserved seat, the seat's visual reservation signal indicates that the pedestrian can sit down there.

With our SUOs we seek to enhance safety for older pedestrians who often have physical impairments and reduced resilience. The SUOs rely on detection of the older pedestrians via Bluetooth technology and provide output with LEDdisplays. The SUOs are implemented with Raspberry Pi.

We conducted scenario based functional testing to validate whether our SUOs are feasible with standard Bluetooth technology. The SUOs have be installed at a major event in a larger German city and have been been positively tested with respect to its functionality in the target environment. 
TABLE I

CONTRIBUTION TO SAFETY WITH SUOS

\begin{tabular}{lll}
\hline Hazardous pedestrian state & Worst-case environmental condition & $\begin{array}{l}\text { Contribution of SUO in avoiding the coincidence of hazardous } \\
\text { pedestrian state and worst-case environmental condition }\end{array}$ \\
\hline Inability to overcome structural barriers & Structural barrier on passage & SUO adaptively point in the direction of an accessible passage. \\
Pedestrian doesn't recognize obstacles & Stumbling block in pedestrian's proximity & SUO adaptively indicates the existance of a stumbling block. \\
Pedestrian needs a seat rest & No awareness on seat availability & $\begin{array}{l}\text { Reservation of a seat from anywhere is possible. } \\
\text { Adaptive signs guide the pedestrian to the reserved seat. }\end{array}$ \\
& & \\
\hline
\end{tabular}

\section{ACKNOWLEDGEMENT}

This work has been supported by the Federal Ministry of Education and Research, Germany, under grant 16SV7438K.

\section{REFERENCES}

[1] OECD, Ed., Ageing and Transport. Paris: OECD, 2001.

[2] J. D. Moreland, J. A. Richardson, C. H. Goldsmith, and C. M. Clase, "Muscle Weakness and Falls in Older Adults: A Systematic Review and Meta-Analysis," Journal of the American Geriatrics Society, vol. 52, no. 7, pp. 1121-1129, 2004. doi: 10.1111/j.1532-5415.2004.52310.x

[3] N. D. Carter, P. Kannus, and K. M. Khan, "Exercise in the Prevention of Falls in Older People," Sports Medicine, vol. 31, no. 6, pp. 427-438, 2001. doi: 10.2165/00007256-200131060-00003

[4] A. M. Bastos, C. G. Faria, E. Moreira, D. Morais, J. M. Melo-de Carvalho, and M. C. Paul, "The importance of neighborhood ecological assets in community dwelling old people aging outcomes: A study in Northern Portugal," Frontiers in Aging Neuroscience, vol. 7, p. Article 156, 2015. doi: 10.3389/fnagi.2015.00156

[5] J. D. Fortuijn, M. van der Meer, V. Burholt, D. Ferring, S. Quattrini, I. R. Hallberg, G. Weber, and G. C. Wenger, "The activity patterns of older adults: a cross-sectional study in six European countries," Population, Space and Place, vol. 12, no. 5, pp. 353-369, 2006. doi: 10.1002/psp.422

[6] Z. Gabriel and A. Bowling, "Quality of life from the perspectives of older people," Ageing and Society, vol. 24, no. 5, pp. 675-691, 2004 doi: $10.1017 / \mathrm{S} 0144686 \mathrm{X} 03001582$

[7] J. Leukel, B. Schehl, S. Wallrafen, and M. Hubl, "Impact of IT Use by Older Adults on Their Outdoor Activities," in Proc. of the 38th Int Conf. on Information Systems (ICIS 2017), 2017.

[8] T. Haustein, J. Mischke, F. Schönfeld, and I. Willand, Eds., Older people in Germany and the EU. Wiesbaden: Federal Statistical Office, 2016.

[9] M. Rantakokko, S. Iwarsson, M. Kauppinen, R. Leinonen, E. Heikkinen, and T. Rantanen, "Quality of Life and Barriers in the Urban Outdoor Environment in Old Age," Journal of the American Geriatrics Society, vol. 58, no. 11, pp. 2154-2159, 2010. doi: 10.1111/j.15325415.2010.03143.x

[10] T. Sugiyama and C. W. Thompson, "Environmental Support for Outdoor Activities and Older People's Quality of Life," Journal of Housing For the Elderly, vol. 19, no. 3-4, pp. 167-185, 2006. doi: 10.1300/J081v19n03_09

[11] F. Bellotti, E. Ferretti, and A. De Gloria, "Discovering the European Heritage Through the ChiKho Educational Web Game," in Proc. of the 1st Int. Conf. on Intelligent Technologies for Interactive Entertainment (INTETAIN 2005). Berlin, Heidelberg: Springer, 2005, pp. 13-22.

[12] E. S. Poulsen, A. Morrison, H. J. Andersen, and O. B. Jensen, "Responsive lighting," in Proceedings of the 15th international conference on Human-computer interaction with mobile devices and services MobileHCI '13. ACM Press, 2013. doi: 10.1145/2493190.2493218 p. 217.

[13] P. Cremonesi, A. D. Rienzo, and F. Garzotto, "Personalized interactive public screens," in Proc. of the 4th Workshop on Interacting with Smart Objects (at ACM IUI 2015), 2015, pp. 10-15.

[14] J. Yang, J. Portilla, and T. Riesgo, "Smart parking service based on Wireless Sensor Networks," in IECON 2012 - 38th Annual Conference on IEEE Industrial Electronics Society. IEEE, 2012. doi: 10.1109/IECON.2012.6389096 pp. 6029-6034.

[15] R. J. Shepard, "Age and Physical Work Capacity," Experimental Aging Research, vol. 25, no. 4, pp. 331-343, 1999. doi: 10.1080/036107399243788
[16] U.S. Department of Transportation, Improving Transportation for a Maturing Society. Washingston, DC: Office of the Assistant Secretary for Transportation Policy, 1997.

[17] N. G. Leveson, Engineering a Safer World. Massachusetts: The MIT Press, 2012.

[18] A. Rayes and S. Salam, Internet of Things From Hype to Reality. Cham: Springer International Publishing, 2019.

[19] L. Atzori, A. Iera, and G. Morabito, "The Internet of Things: A survey," Computer Networks, vol. 54, no. 15, pp. 2787-2805, 2010. doi: 10.1016/j.comnet.2010.05.010

[20] L. D. Xu, W. He, and S. Li, "Internet of Things in Industries: A Survey," IEEE Transactions on Industrial Informatics, vol. 10, no. 4, pp. 2233 2243, 2014. doi: 10.1109/TII.2014.2300753

[21] G. Kortuem, F. Kawasar, D. Fitton, and V. Sundramoorthy, "Smart objects as building blocks for the internet of things," IEEE Internet Computing, vol. 14, no. 1, pp. 44-51, 2010. doi: 10.1109/MIC.2009.143

[22] T. S. López, D. C. Ranasinghe, B. Patkai, and D. McFarlane, "Taxonomy, technology and applications of smart objects," Information Systems Frontiers, vol. 13, no. 2, pp. 281-300, 2011. doi: 10.1007/s10796-0099218-4

[23] J.-P. Vasseur and A. Dunkels, "Smart Cities and Urban Networks," in Interconnecting Smart Objects with IP. Burlington: Elsevier, 2010, ch. 22, pp. 335-351.

[24] B. Hammi, R. Khatoun, S. Zeadally, A. Fayad, and L. Khoukhi, "IoT technologies for smart cities," IET Networks, vol. 7, no. 1, pp. 1-13, 2018. doi: 10.1049/iet-net.2017.0163

[25] A. Zanella, N. Bui, A. Castellani, L. Vangelista, and M. Zorzi, "Internet of Things for Smart Cities," IEEE Internet of Things Journal, vol. 1, no. 1, pp. 22-32, 2014. doi: 10.1109/JIOT.2014.2306328

[26] A. Huldtgren, C. Katsimerou, A. Kuijsters, J. A. Redi, and I. E. J. Heynderickx, "Design Considerations for Adaptive Lighting to Improve Senior's Mood," in Proceedings of the 13th International Conference on Smart Homes and Health Telematics (ICOST 2015). Springer (LNCS), 2015. doi: 10.1007/978-3-319-19312-0_2 pp. 15-26.

[27] M. Cunha and H. Fuks, "AmbLEDs: Implicit I/O for AAL Systems," in Proceedings of the 4th Workshop on Interacting with Smart Objects (at ACM IUI 2015), 2015, pp. 6-9.

[28] J. Müller, F. Alt, D. Michelis, and A. Schmidt, "Requirements and design space for interactive public displays," in Proceedings of the international conference on Multimedia - MM '10. New York, New York, USA: ACM Press, 2010. doi: 10.1145/1873951.1874203 p. 1285.

[29] D. Vogel and R. Balakrishnan, "Interactive public ambient displays," in Proceedings of the 17th annual ACM symposium on User interface software and technology - UIST '04. ACM Press, 2004. doi: 10.1145/1029632.1029656 p. 137.

[30] M. Traunmueller and A. Fatah gen. Schieck, "Introducing the space recommender system," in Proceedings of the 6th International Conference on Communities and Technologies - C\&T'13. ACM Press, 2013. doi: 10.1145/2482991.2482995 pp. $149-156$

[31] S. Foell, G. Kortuem, R. Rawassizadeh, M. Handte, U. Iqbal, and P. Marrón, "Micro-Navigation for Urban Bus Passengers: Using the Internet of Things to Improve the Public Transport Experience," in Proceedings of the The First International Conference on IoT in Urban Space. ICST, 2014. doi: 10.4108/icst.urb-iot.2014.257373 pp. 1-6.

[32] P. M. Kumar, U. Gandhi, R. Varatharajan, G. Manogaran, J. R., and T. Vadivel, "Intelligent face recognition and navigation system using neural learning for smart security in Internet of Things," Cluster Computing, 2017. doi: 10.1007/s10586-017-1323-4 